\title{
Application of IoT for Power Controlling in Smart Home
}

\author{
Muhammad Rauf \\ Electronic Engineering Department, \\ Dawood University of Engineering and \\ Technology, Karachi \\ muhammad.rauf@duet.edu.pk
}

\author{
Abid Muhammad Khan \\ Electrical Engineering Department, Sir \\ Syed University of Engineering and \\ Technology, Karachi \\ abidmk@ssuet.edu.pk \\ Qazi Raza Ali \\ Electronic Engineering Department, Sir \\ Syed University of Engineering and \\ Technology, Karachi \\ raza5858@gmail.com
}

\author{
Atif Shaikh \\ Department of Computer System \\ Engineering, Dawood University of \\ Engineering and Technology, Karachi \\ atif.jamil@duet.edu.pk
}

\begin{abstract}
The rapid growth in the population of major cities is causing an increase in energy consumption. Several approaches, including the use of recent technological advancements, are becoming increasingly important. IoTbased applications are becoming more common in addressing a variety of real-time issues. The central idea of the project in collaborating with this new technology is the human-machine interaction. Furthermore, the proposed architecture enables energy-saving applications that perform the following primary functions: estimating energy utilization of the home environment using metering devices, and applying user-defined limits that cut off boundaries providing if it exceeds energy limits. The Internet serves as an interactive medium between servers and home appliances, monitoring and controlling energy consumption remotely. For this purpose, the android platform is used to design a mobile application. Raspberry $\mathrm{Pi}$ is dedicated to the server that maintains the database of each controllable unit. The proposed idea is successfully tested in the laboratory environment with synthetic load and in real-time over a running energy meter, to evaluate the efficiency and effectiveness.
\end{abstract}

Keywords- Energy monitoring, Wifi module, cloud server, smart home, IoT

\section{INTRODUCTION}

Worldwide computer networks have paved the way for broad, intelligent and wireless interaction between the objects. The need for everything-to-everything connectivity gave rise to terms called internet of things (IoT). IoT allows objects to be smart and to be accessed remotely across existing network infrastructure [1]. The focused applications of IoT are mainly the smart homes, telemedicine, and industrial domain [2]. Furthermore, the integration of Wireless Sensor Network (WSN) and IoT have enabled a broader interconnection of virtual and physical entities for the given applications. In recent years, the steep advancement in network interacting capability and electrical communication systems have inspired the system development of the home-automated things. This method is used to track or monitor a condition that is crucial in daily energy utilization. A realtime navigation of a resident's utility consumption in a house is an example of such a method [3]. Utility bills paid online are incorporating the aspects of information technology. These energy monitoring advancements have lead the ways for future energy saving plans [4]. However, assessment and monitoring of energy meter values, interpretation, and appliance control are not well integrated. Revenue loss occurs as well. AMR (Automated Meter Reading) is one of the innovations that integrates automatic billing and payment processing. AMR requires allocation of IP addresses to each energy unit meter [5]. A network of interconnected objects connected to the internet for the application of smart homes is depicted in Fig 1. Utilizing IoT-based technologies has brought with it an increased focus on efficient power utilization. There are many approaches to cut energy use. The options may include various energy information such as working states of devices and power usage for customers with the help of electric and digital meters. Also, providing energy-saving tips for customers is part of the service [6].

In the related field, various studies have been published in which an IoT-based system is presented. AMR-based system is built to detect appliance energy consumption in general. The sensor for measuring the optical energy produced by the LED was used in the design. The optical response provides the energy usage and then the microcontroller controls further [7,8]. Their objective is to design a model that facilitates hassle-free, quick, and portable internet connectivity for the user. It is still challenging to implement an energy-saving system in real time. First, it aims to combat human behavior when it comes to using energy through commonly found home appliances. The proposed model includes a remote sensing device, wireless actuators, sensors, and a webbased interface for computers and mobile applications. Finally, by confronting the model's power, the energy 
usage can be manipulated either automatically or manually, and even switching on or switching off certain devices according to their tariff plan [9].



Fig. 1 Overview of IoT based smart home systems

This proposed work is aimed at providing a wide array of services where, even in standby mode, the appliance continues to be capable of maintaining its communication capacity. The network will be able to analyze its own status and determine its current state. According to the author's understanding, the concept has yet to be implemented on energy-consuming equipment. The proposed system can be developed using a wide range of devices, including Node MCU (Microcontroller Unit) [10] and Arduino UNO clones like Espduino [11] and Wemos D1 [12]. Many works have been published on the topic of using ESP8266 modules. The above domain of application is widespread, encompassing both distributed home automation systems [13] and several other applications including smart water management systems [14] and vibration sensor networks [15] etc. Extending the application for a low cost, efficient, and flexible energy monitoring and controlling system, this paper is mainly interested in maintaining a wide application. A residential area cloud-server communications integration system consists of a SoC (System on Chip), which includes the smart meeting application, and a cloud server.

The rest of the article comprises of section 2 as methodology where the architecture of the design and flow chart of the work is focused. Section 3 elaborates the implementation phases whereas section 4 present the results and discussion. Lastly the conclusion is provided.

\section{METHODOLOGY}

\section{A. System Overview}

The main goal of the proposed work was to track, monitor and store the status data of home-related devices and related sensors controlled by a cloud-based data server gateway. The system's topology and associated connectivity are depicted in this section. The proposed scheme consists of a smart identifying unit, wireless sensors and actuators, as well as a web-based platform for remote and mobile application development. The system as a whole can be divided into two domains. The first is the home interconnection, which monitors and transmits data from dedicated appliances and associated sensors to the data server. The second component is the cloud-based data server, which organizes received data and provides services to users by transmitting appropriate signals and receiving user commands for control purposes. Such monitoring and control mechanisms have the potential to advance residential unit energy efficiency.

\section{B. Architecture}

The proposed system is divided into three sections that are mentioned below.

1) Customer Domain: The project consists of an MCU, energy meter, optical sensor and ESP8266 at the customer side. The sensor is used to detect the falling edge of the LED that is mounted on the electric meter. The Wifi router attached to the customer domain is configured with the internet. Subsequent to finalize the authentication procedure, the consumption information is transmitted to the server system.

2) Server and Control: Following that, the received data is tabulated and stored in the designated variable. The core server sends the received data to the unit consumption server and notifies the users. It is also in charge of all other system-related functionality. By providing messaging services, the app server acts as a conduit between the user and the main server.

3) Customer Interface: Web-based mobile app is selected as the messaging application for sending queries and problems to the core server. The user's requests are routed to the main server, which responds in predefined ways. Figure 2 depicts the process in greater detail.

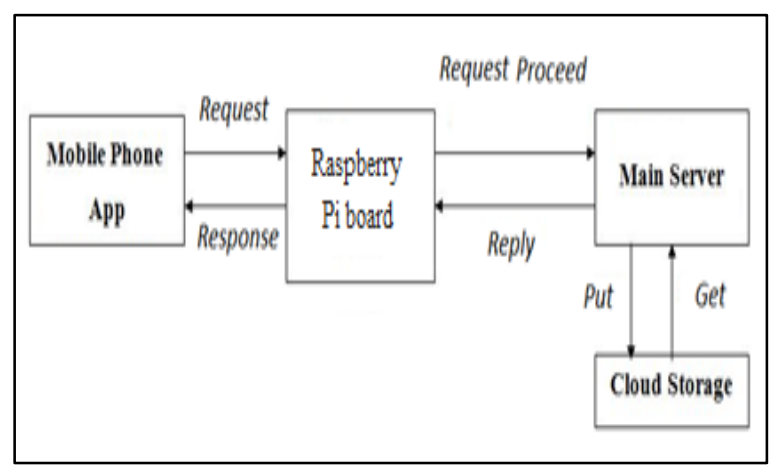

Fig. 2 Proposed Baseline Architecture 


\section{Implementation Flow}

This section details the process flow of the proposed work related to the sensor interfacing as shown in Fig. 3.

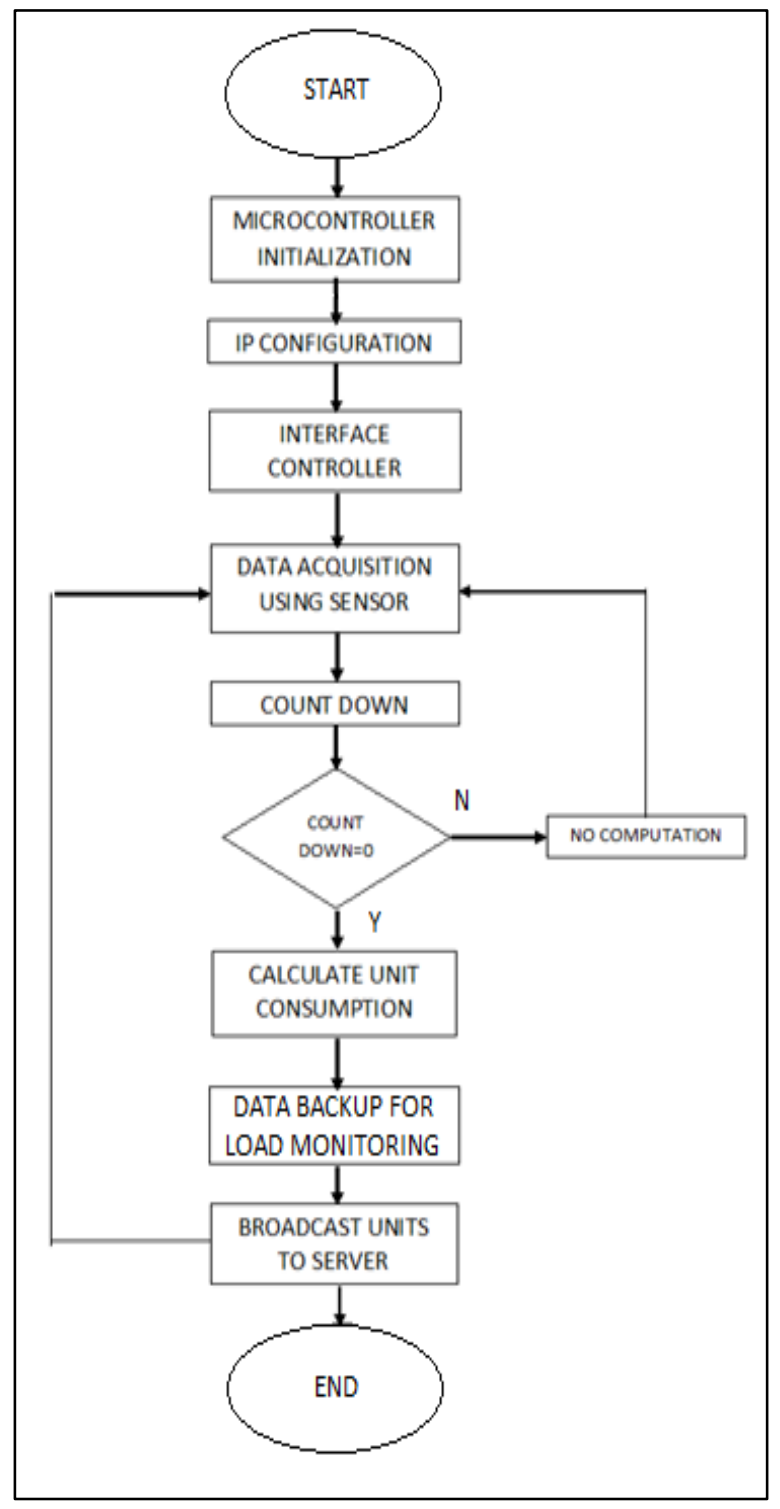

Fig. 3 Proposed Flow Chart

Before associating the controller with the wireless sensor, the controller is first initialized, after which it is paired with the wireless sensor and assigned a password. The energy meter is now equipped with an IP address and is ready to help guide data used over the internet. The controller is now capable of sending the data packets to the cloud storage following the authorization steps that involve transferring tokens. In addition, the LED toggling on the energy meter is used to convert the digital waveform to an analogue voltage signal which is interpreted by the MCU as depicted in Fig 4.

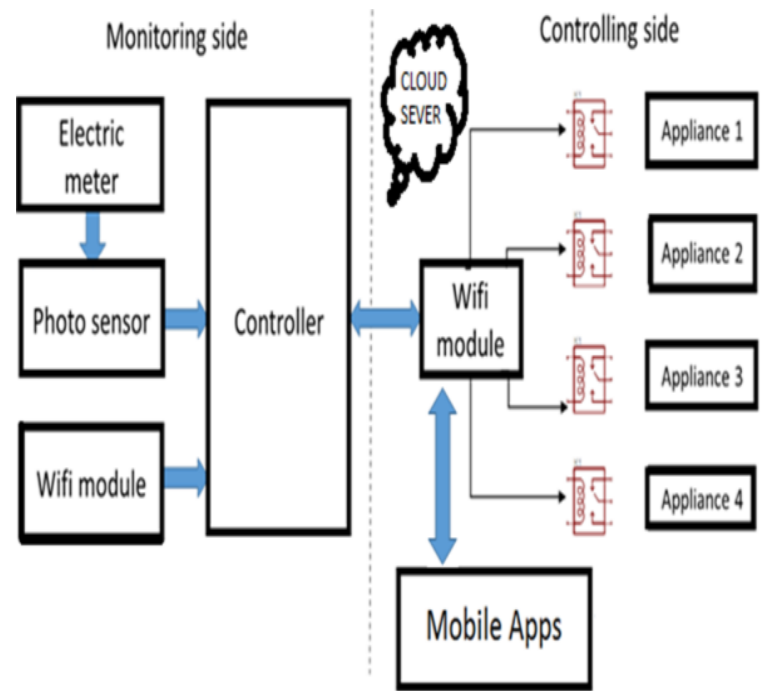

Fig. 4 Proposed Block Diagram of Project

Once the interrupt routine has calculated the energy unit consumed, the routine utilizes the voltage pulse generated by the sensor to calculate the energy unit consumed. The computed unit is updated with the previously accumulated unit in the cloud every time a pulse is received. Sensing updates the process automatically.

The Controller is capable of offering customizations, as well as handling power cuts, through various stages. PC's and Android applications also enable different appliances to be managed. An SSID and password can be configured using our Wi-Fi module. The sensor tells the appliance how much is being used, and the LCD display shows this value. In addition, a cutoff boundary has been assigned, on which power is turned off and appliances stop functioning.

\section{Hardware Design}

This section explains the reason for constructing a network that utilizes IEEE 802.11 wireless technology and ESP8266 cheap Wi-Fi communication modules, both of which are already widely available. First, the linkgenerating circuits are tested in advance to guarantee authentic and reliable results.

The ATMEGA 328 is the type of ATMEGA328 microcontroller that enables the integration and connection of electronic devices. Unlike relays, transistors are versatile, offering designer's greater flexibility in redesigning the prototype to accommodate various applications.

\section{IMPLEMENTATION PHASES}

The implementation phases of the proposed idea is given as: 


\section{A. Initiation}

All project requirements are concentrated in this phase, which includes electricity metering systems that are already in place. The first stage of this three-stage process is called defining project objectives, scope, and phases. This research takes place in a simple model house that has room lights and fans among other appliances, including switches.

\section{B. Planning}

The project development strategy, resource allocation, and techniques to link the system with implemented meter-based systems are all part of the planning phase. Additionally, project scopes are defined where three AC appliances and two rooms are involved, as well as an energy meter is planned to be utilized in the study.

\section{Designing and Debugging}

The steps involved in it are circuit design, server development, and debugging. The prior study parameters, particularly those listed in [7] of the project, are included and used in the project's development. The first project is solely focused on optocoupler integration. Following then, a single load appliance is monitored and controlled, and only then is the project tested for multiple loads. A prototype is created throughout the way, which is then used to acquire the outcomes.

\section{Design Analysis}

The analysis is carried out on several days and times once the project design has been tested. Weekdays and weekends were chosen so that trustworthy findings could be obtained. In order to achieve the final analyses of the developed prototype, several sets of time are also taken into account. The suggested model's whole functionality is made available on an internet platform that may be accessed by any equipment with a network connection. An android application for monitoring and management has also been built.

A particular IP address and user name and password have been assigned to the user, who can then use these credentials to access the webpage and manage all of their appliances from a remote location. The menu allows the user to easily manage the various appliances that are present. Any user with a given IP address, id, and password can easily access the designed web page, but hardware installation is required to fully utilize the technology.

When the user enters the login page, he will be able to access his confidential area, where the server will be able to fully gather the home conditions and total power utilization of each appliance. Within the menu, the owner can navigate between the various devices and manually switch some of them as needed.
Figure 5 depicts the primary level software-based command and control system. The design of the server interface and app is inspired from [6].

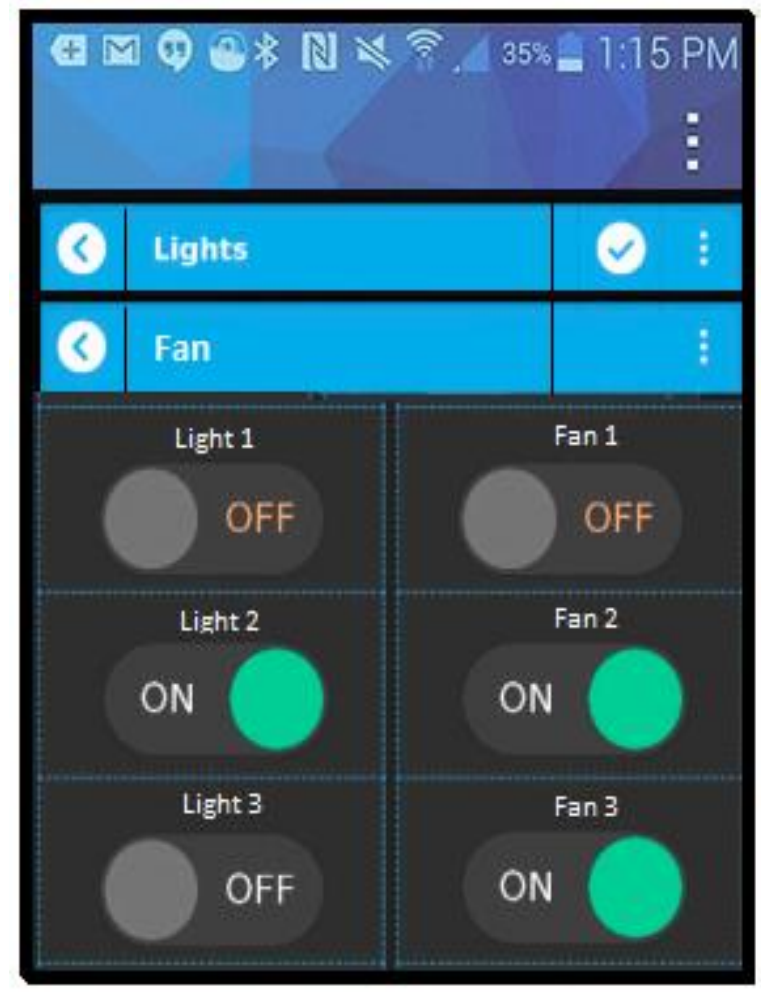

Fig. 5 The depiction of Mobile Server

\section{RESULTS AND DISCUSSION}

The system has been specifically designed to monitor the appliances, identify their energy-using patterns, and then adjust them on the basis of the information obtained. The important feature of a project is the efficiency of the monitoring system that's put in place at the home.

A running energy meter is noted for three days, which is done so that its units can be calculated for the purpose. Since there are a lot of unpredictable activities to be done in these days, the days are chosen to be active. At the halfway point between two hour intervals, the customer units are observed.

It is possible to note a possible trend in electricity consumption in the reading. Once the optocoupler part of the project is designed and installed on the meter, the project moves forward with the design and implementation of the system. Because these readings will be further required to control the home appliances, these readings demonstrate the system's dependability.

Figure. 6 presents the measurement of the input voltage received by the house. 


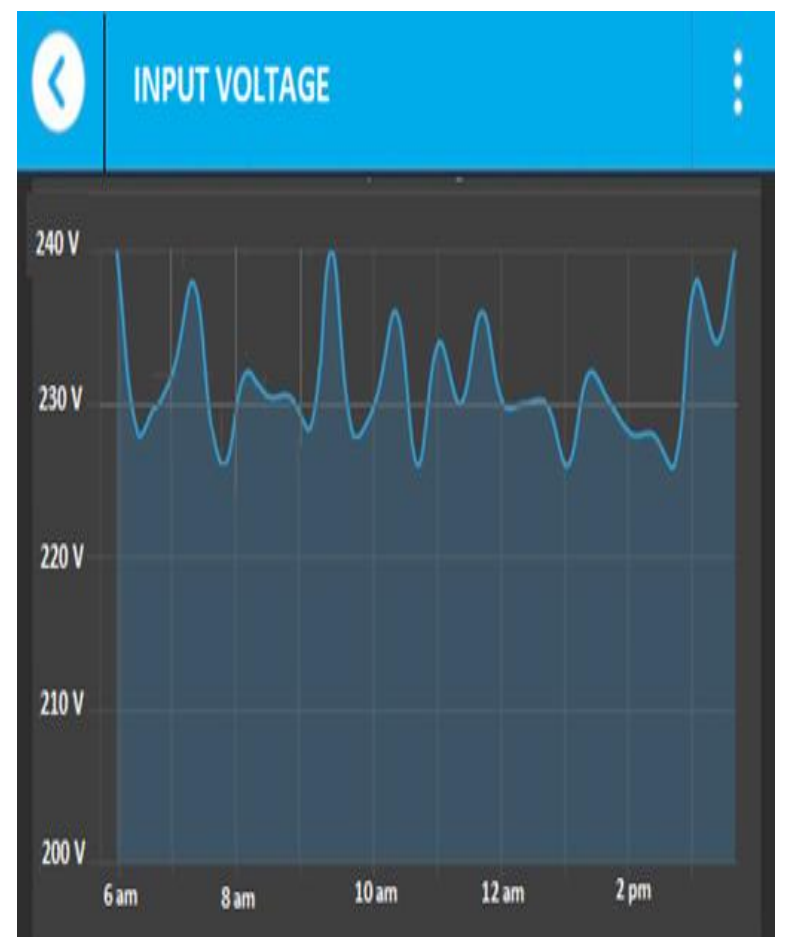

Fig. 6 A depiction of power monitoring using input voltage to the house

It will be further used to calculated power. In the image as given in Fig 7, it is seen that the reading for the manual meter is appeared.

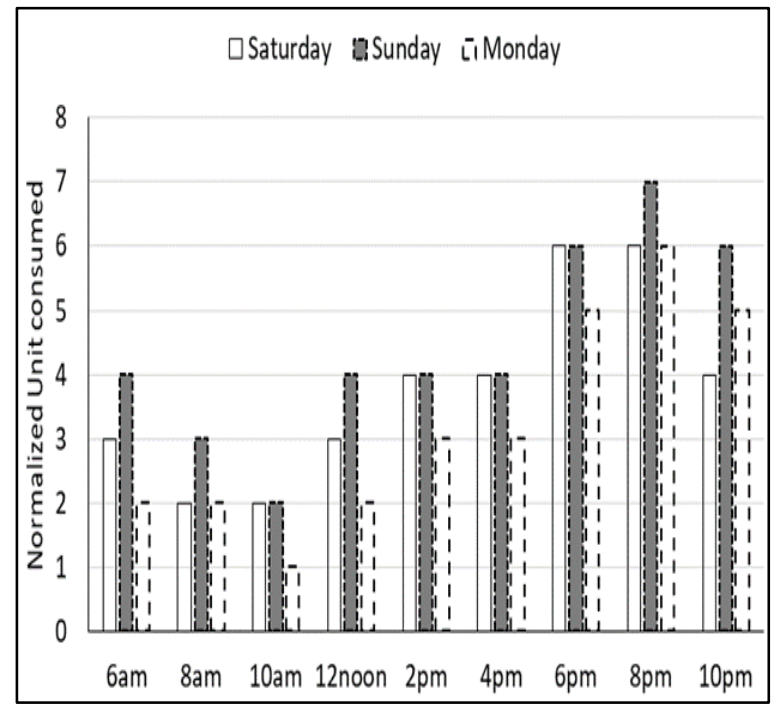

Fig. 7 Manually noted Electricity unit consumed for three days at the interval of 2 hours

In the image as shown in Fig 8, one can see that there is a minor deviation from the design as shown by the readings that were manually noted.
Figure 8 demonstrates unequivocally that the deviation between manual and measured values is a maximum of two units and a minimum of one unit per day.

To say that the data collected for monitoring and controlling is reliable is to indicate that the data is trustworthy. Implementation is the next stage. The design is put in place, as per the plan shown in Figure 3.The user was able to monitor his house's units consumed from anywhere because of the project's successful implementation. It's estimated that the $20-30 \%$ reduction in units is possible.

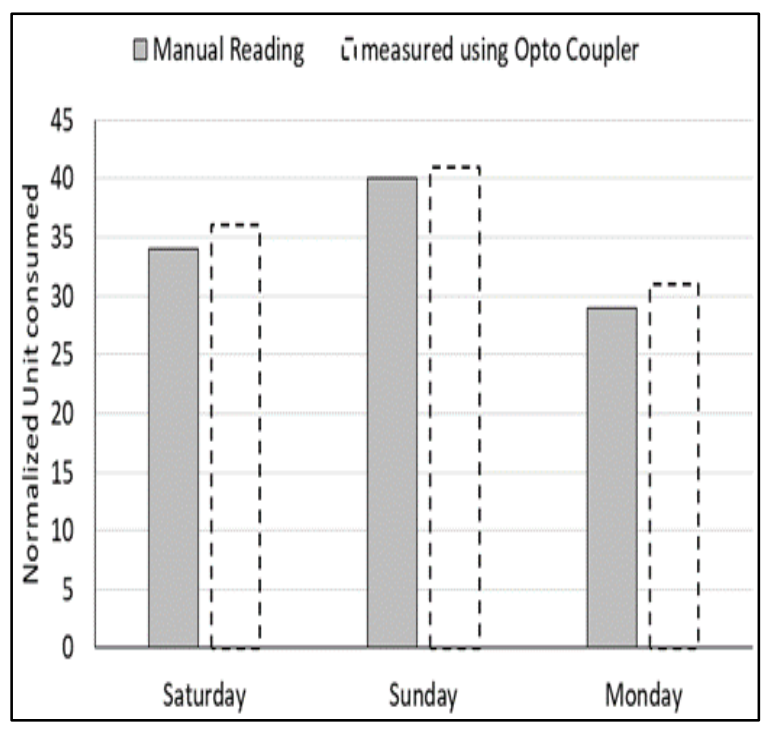

Fig. 8 Comparison of manual and measured electricity units consumed

\section{CONCLUSION}

This project aims to create an energy-efficient ad-hoc MAC protocol-based wireless network with static modules. This smart home model connects various electrical appliances in the home via an MCU and enables them to be monitored and controlled with little or no manual intervention. Additionally, on the meter side, a low-cost single board capable of sensing, processing, and transmitting real-time energy data is developed. Additionally, it is capable of providing a user-friendly platform for cloud storage customers.

While the prototype demonstrated promising results, the proposed scheme's implementation is contingent on a variety of environmental and human factors, including the operation of the selected hardware, the specific data types, and the electrical infrastructure. Thus, additional research will undoubtedly be required in the future to determine the appropriate implementation scenarios for this scheme in conjunction with other relevant factors. Additionally, the effects of extremely high node densities must be investigated, as well as methods for planning the proposed scheme at the transport level. 


\section{REFERENCES}

[1] E. S. A. Ahmed, "Internet of things applications, challenges and related future technologies," World scientific news, vol. 67, no. 2, pp. 126-148, 2017.

[2] El-hajj, M., Fadlallah, A., Chamoun, M. and Serhrouchni, A. A Survey of Internet of Things (IoT) Authentication Schemes. Sensors, 19(5), p.1141, 2019.

[3] R. Majeed, N. A. Abdullah, I. Ashraf, Y. B. Zikria, M. F. Mushtaq, and M. Umer, "An Intelligent, Secure, and Smart Home Automation System," Scientific Programming, vol. 2020, p. 4579291, 2020.

[4] D. Alahakoon and X. Yu, "Smart Electricity Meter Data Intelligence for Future Energy Systems: A Survey," IEEE Transactions on Industrial Informatics, vol. 12, no. 1, pp. 425436, 2016.

[5] Q. M. Ashraf, M. I. M. Yusoff, A. A. Azman, N. M. Nor, N. A. A. Fuzi, M. S. Saharedan, and N. A. Omar, "Energy monitoring prototype for Internet of Things: Preliminary results," 2015 IEEE 2nd World Forum on Internet of Things (WF-IoT), 2015.

[6] Y.-H. Lee, "Context-aware energy consumption analysis and energy savings system," Master's thesis, Department of Computer Science and Information Engineering, College of Electrical Engineering and Computer Science, National Taiwan University, July 2009.

[7] A. Ali, N. A. Razali, N. H. Saad, N. Vitee, "Implementation of Automatic Meter Reading (AMR) Using Radio Frequency (RF) Module in IEEE International Conference on Power and Energy (PECon), 2012.

[8] A. Fakharuddin, A. N. Abdalla, M. Rauf, N. M. Kamil, S. Ahmad, and A. Mustafa. (2012). "A smart energy management system for monitoring and controlling time of power consumption", Scientific Research and Essays, 7(9), 1000-1011, 2012.

[9] M. Darianian and M. P. Michael, "Smart home mobile RFID based internet-of-things systems and services," in Proceedings of the 2008 International Conference on Advanced Computer theory and Engineering, pp. 116-120, IEEE, 2008.

[10] A. Zarzycki, "Adaptive Designs with Distributed Intelligent Systems - Building Design Applications," - Proceedings of the 34th International Conference on Education and Research in Computer Aided Architectural Design in Finland, pp. 24.-26, Towards Smarter Cities Concepts and Strategies - Volume 1 eCAADe 34, 2016.

[11] M. Wang, R. Zhang, Y. Zhang, H. Dong and Y. Wang, "An Indoor Environmental Monitoring system Based on ESPDuino for Green Buildings," 2018 IEEE International Conference on Information and Automation (ICIA), pp. 1658-1663, 2018.

[12] Wemos D1 - official site, https://www.wemos.cc/product/d1.html. Retrieved December 2020.

[13] T.A. Abdulrahman, O.H. Isiwekpeni, N.T. Surajudeen-Bakinde, A.O. Otuoze. "Design, Specification and Implementation of a Distributed Home Automation System," Procedia Computer Science, Volume 94, 2016, pp. 473-478, ISSN 1877-0509, 2016.

[14] K. M. Shahanas, P. B. Sivakumar, "Framework for a Smart Water Management System in the Context of Smart City Initiatives in India," Procedia Computer Science, Volume 92, 2016, pp. 142147, ISSN 1877-0509, 2016.

[15] S. Biansoongnern, B. Plungkang, S. Susuk, "Development of Low Cost Vibration Sensor Network for Early Warning System of Landslides," Energy Procedia, Volume 89, 2016.

[16] C. Stolojescu-Crisan, C. Crisan, and B.-P. Butunoi, "An IoTBased Smart Home Automation System," Sensors, vol. 21, p. 3784,2021 